\title{
The frequency equation of Rayleigh waves in a piezoelectric smart material
}

\author{
Abo-el-nour Abd-alla and Fatimah Alshaikh
}

\begin{abstract}
The frequency equation of the Rayleigh wave propagating in an anisotropic smart piezoelectric material is obtained. The non-dimensional velocity of the Rayleigh wave is computed for Barium Titanate. The surface mechanical displacements and electric fields are found as a function of layer thickness and are presented graphically. This theoretical work may be helpful in further experimental works on surface wave propagation in piezoelectric materials and surface acoustic wave filter devices.
\end{abstract}

Keywords-Rayeigh wave, frequancy equation, Piezoelectric material, Barium Titanate.

\section{Introduction}

In recent years, piezoelectric materials have been increasingly applied to different engineering structures, especially to smart or intelligent systems as intelligent sensors, damage detectors, etc. Rayleigh waves are the most important of the surface acoustic waves which are essentially considered as mechanical waves. Surface Acoustic Waves (SAWs) were first explained in 1885 by Lord Rayleigh, who described the surface acoustic mode of propagation and predicted its properties in his classic paper [1].

Basic discoveries of piezoelectricity were made in the late 19th century by Pierre and Paul-Jacques Curie [2]. After that, there has been a tremendous growth in the telecommunications industry over the past few decades with a subsequent increase in demand for high-quality components which were based on the piezoelectricity phenomenon. In particular, usage of SAW devices in wireless communication systems demands higher operating frequencies, wider bandwidths, smaller sizes and lower insertion losses. Consequently, there is a pressing need for improved techniques for device fabrication and response prediction. Further development of photolithographic techniques for computer chips and telecommunication devices to optically transfer micro- and nanostructure patterns onto a substrate, allowed fabrication of micro- and nanostructures implemented in modern biosensors [3].

There are many studies that focused on investigating Rayleigh waves in different anisotropic media without piezoelectric effects such as [4] and [5]. While, using a different method or additional fields such as the thermal field and/or magnetic field, many authors obtained explicit Rayleigh wave speeds for isotropic materials, e.g. [6], [7] and [8]. The anisotropic behavior of the medium extremely modifies the existence and the structure of the SAW and BAW that propagates at the free surface of the

Department of Mathematics, Faculty of Science, Jazan University, Jazan, Saudi Arabia medium [9] and [10]. Anisotropy, therefore, induces considerable difficulties in analytically and explicitly studying wave propagation. Attempts to derive explicit secular equations have been reported in [11] and [12]. In seismology, Rayleigh waves cause destructive vibration to the structures. However, due to nonlinearity, it is difficult to get the exact solution of the characteristic equation of Rayleigh waves in anisotropic media [13]. Lately, The propagation of surface Rayleigh waves in a half-space under the effect of pre-stress was examined by many authors such as: [14] and [15]. The piezoelectric materials (are called smart materials) are capable of altering the structure's response through sensing, actuation and control [16] and [17]. Piezoelectricity may be used in many other useful different applications for examples see, [18] and [19]. The nonlinear constitutive equations for magnetoacoustic or thermo-electro-elastic materials which are considered the basic tools for studying the propagation of surface acoustic waves in isotropic or anisotropic materials may be found in many works of literature such as [20]-[25].

Recently, There are several papers concerning various problems based on the theory of surface acoustic waves, especially, a Rayleigh surface waves propagation problem in many hypotheses (see, e.g., Chirita et al. [26], Sharma [27], Bucur et al. [28], Torello et al. [29], Lin et al. [30] and Singh [31].

The present paper investigates Rayleigh surface acoustic waves in anisotropic piezoelectric materials. A details solution giving plots for variations of fields and displacements as a function of distance from the free surface are obtained. The secular equation under suitable mathematical boundary conditions of the wave motion is derived. The characteristics of surface waves propagating in piezoelectric elastic half-space and their dependence upon the physical parameters are investigated.

\section{2- The basic equations}

In the quasi-static approximation, the governing field equations of piezoelectricity can be expressed as [23]:

$$
T_{i j, j}=\rho \ddot{u}_{i}
$$

Propagation on piezoelectric crystals is usually complicated by the presence of a piezoelectric surface wave which contains electromagnetic field quantities. These field quantities must satisfy Maxwell's equations for magnetically isotropic dielectric [22],

$\varepsilon_{i j k} E_{k, j}=-\mu_{\circ} \dot{H}_{i}, \varepsilon_{i j k} H_{k, j}=\dot{D}_{i}, H_{j, j}=0, D_{i, i}=0$ (2) A combination of Eqs. (2) 1 and (2) 2 which eliminates the magnetic field, $H_{i}$, is of particular use here,

$$
E_{j, i j}-E_{i, j j}=-\mu_{\circ} \ddot{D}_{i} \text {. }
$$

Where the definition of all the symbols are written in Appendix B. In a piezoelectric material, the mechanical equations of motion and Maxwell's equations for the 
electrical behavior are coupled through two constitutive equations given by:

- The stress tensor for piezoelectric materials is:

$T_{i j}=C_{i j k \ell} S_{k \ell}-e_{k i j} E_{k}$,

- The components of the electric displacement are given by: $D_{i}=\varepsilon_{i k} E_{k}+e_{i k \ell} S_{k \ell}$.

- The strain tensor is related to the displacements, $u_{i}$, by:

$S_{i j}=\frac{1}{2}\left(u_{i, j}+u_{j, i}\right)$.

Elastic and piezoelectric wave propagation for piezoelectric crystals can be summarized as a solution of equations of motion (1) with constitutive relation (2) subjected to the following boundary conditions:

(i) The surface of propagation is stress-free,

(ii) The electromagnetic field components have appropriately matched the surface.

For simplicity, engineering notation will now be employed to reduce the number of subscripts. Engineering notation allows designation of elastic constants by two subscripts, stress components by one and similar reductions of other appropriate quantities.

Solutions of interest are those that describe surface wave propagation. A nonuniform plane wave solution will be assumed with propagation in one coordinate direction, attenuation in another, and independence in the third. Examination of equations (4) and (1) indicates the optimum choice to be in the $x_{3}$ direction, attenuation in the $x_{2}$ direction and no variation in the $x_{1}$ direction. solutions of the following form are assumed for a crystal occupying the half-space $x_{2} \leq 0$,

$$
\begin{aligned}
& u_{j}=A_{j} \exp \left[i\left\{k\left(\alpha x_{2}+x_{3}\right)-\omega t\right\}\right], \\
& E_{j}=k B_{j} \exp \left[i\left\{k\left(\alpha x_{2}+x_{3}\right)-\omega t\right\}\right],
\end{aligned}
$$

where $j=1,2,3, \omega$ is the angular frequency, $A_{j}, B_{j}$ are unknown arbitrary amplitude constants, $k, \alpha$ are the wave number and a dimensionless decay constant respectively, that to be determined and $\mathrm{t}$ is the time. $E_{j}$ amplitudes include the factor of $k$ for later convenience.

Substitution of Eqs. (7) successively into Eqs. (6), (4), (1) and (3) gives the following homogeneous system of equations for surface wave propagation:

$$
\left|\begin{array}{cccccc||c}
a_{11} & a_{12} & 0 & 0 & 0 & 0 \\
a_{21} & a_{22} & 0 & 0 & 0 & 0 \\
0 & 0 & a_{33} & a_{34} & a_{35} & a_{36} \\
0 & 0 & a_{43} & a_{44} & a_{45} & a_{46} & A_{1} \\
B_{1} \\
A_{2} \\
A_{3} \\
0 & 0 & a_{53} & a_{54} & a_{55} & a_{56} \\
B_{2} \\
B_{3}
\end{array}\right|=0
$$

where

$$
\begin{aligned}
& a_{11}=-v^{2} \rho+2 C_{14} \alpha+C_{66} \alpha^{2}+C_{44}, \\
& a_{21}=\mu_{0} v^{2} a_{12}=i \mu_{0} v^{2}\left(e_{15}-e_{22} \alpha\right), \\
& a_{22}=\mu_{0} \varepsilon_{11} v^{2}-\alpha^{2}-1, \\
& a_{33}=-v^{2} \rho+C_{11} \alpha^{2}-2 C_{14} \alpha+C_{44}, \\
& a_{43}=a_{34}=C_{44} \alpha+C_{13} \alpha-C_{14} \alpha^{2}, \\
& a_{53}=\mu_{0} v^{2} a_{35}=i \mu_{0} v^{2}\left(e_{22} \alpha+e_{15}\right),
\end{aligned}
$$

$$
\begin{aligned}
& a_{44}=-v^{2} \rho+C_{44} \alpha^{2}+C_{33}, \\
& a_{55}=\mu_{0} \varepsilon_{11} v^{2}-1, \\
& a_{65}=a_{56}=\alpha, \\
& a_{66}=\mu_{0} \varepsilon_{33} v^{2}-\alpha^{2} . \\
& a_{63}=\mu_{0} v^{2} a_{36}=i \mu_{0} v^{2} e_{31} \alpha, \\
& a_{54}=\mu_{0} v^{2} a_{45}=i \mu_{0} v^{2} e_{15} \alpha, \\
& a_{64}=\mu_{0} v^{2} a_{46}=i \mu_{0} v^{2} e_{33},
\end{aligned}
$$

Examination of Eqs. (8) indicates the existence of two uncoupled modes of propagation. One of these contains $u_{1}$ and $E_{1}$ which is described by:

$u_{1} \neq 0, \quad E_{1} \neq 0, \quad u_{2}=u_{3}=E_{2}=E_{3}=0$,

$\left|\begin{array}{ll||l}a_{11} & a_{12} \\ a_{21} & a_{22}\end{array}\right| \begin{aligned} & A_{1} \\ & B_{1}\end{aligned} \mid=0$

This equation contains nonzero components transverse to the direction of propagation and is referred to as the uncoupled transverse mode.

The second solution is described by

$$
\begin{gathered}
u_{1}=E_{1}=0, \quad u_{2} \neq 0, \quad u_{3} \neq 0, \quad E_{2} \neq 0, \quad E_{3} \neq 0, \\
\left|\begin{array}{llll||c}
a_{33} & a_{34} & a_{35} & a_{36} \\
a_{43} & a_{44} & a_{45} & a_{46} \\
a_{53} & a_{54} & a_{55} & a_{56} \\
a_{63} & a_{64} & a_{65} & a_{66} & A_{3} \\
A_{2} \\
B_{3}
\end{array}\right|=0 .
\end{gathered}
$$

Here the nonzero components of displacement and electric field are coplanar, in the plane normal to the surface and parallel to the direction of propagation, such a plane is referred to as the sagittal plane and the mode is referred to as the Rayleigh mode. These two possible modes will be discussed in details later.

\section{Electromagnetic field outside the crystal}

The assumed solutions, Eqs. (7), are valid for the half-space $x_{2} \leq 0$ occupied by the material. The displacement components, $u_{i}$, will certainly be zero outside the material, $x_{2}>0$, but the electromagnetic field will not. To assure matching of the fields along the surface, space and the time dependence of those fields outside must be of the same form as those inside differing by amplitude constants, wave number, and decay constants only. These fields must also obey Maxwell's equations in free space,

$$
E_{j, i j}-E_{i, j j}=-\varepsilon_{\circ} \mu_{\circ} \ddot{E}_{i},
$$

Considering solutions in the form for Eqs. (12) where $\left(x_{2}>0\right)$,

$$
E_{j}=k C_{j} \exp \left[i\left\{k\left(\beta x_{2}+x_{3}\right)-\omega t\right\}\right], \quad(j=1,2,3)
$$

where $\beta$ is the decay constant, $C_{j}$ are the amplitudes and $k$ is the wave number are to be determined. Substituting from Eqs. (13) into (12) produces a set of homogeneous equations which govern the propagation of fields above the surface. replacing the phase velocity, $\omega / k$, by $v$ and expressing in determinant form these equations are given by: 
$\left|\begin{array}{lll||l}a_{11} & a_{12} & a_{13} & C_{1} \\ a_{21} & a_{22} & a_{23} & C_{2} \\ a_{31} & a_{32} & a_{33} & C_{3}\end{array}\right|=0$

where

$a_{11}=-\beta^{2}-1+\varepsilon_{0} \mu_{0} v^{2}, \quad a_{22}=1-\varepsilon_{0} \mu_{0} v^{2}$,

$a_{33}=\beta^{2}-\varepsilon_{0} \mu_{0} v^{2}, a_{12}=0, a_{21}=0, a_{31}=0$

, $a_{13}=0, \quad a_{32}=a_{23}=-\beta$.

This set of equations shows two uncoupled modes; one contains the $E_{1}$ component of the field only, corresponding to the transverse mode, and the second contains $E_{2}$ and $E_{3}$ components representing the Rayleigh mode. From Eq. (14) to obtain a nontrivial solution requires the coefficient determinant to be zero. Therefore, the decay constant $\beta$ is found as

$\beta= \pm i \sqrt{\left(1-\varepsilon_{0} \mu_{0} v^{2}\right)}$

using the boundary conditions requiring fields to vanish at infinity leads to the correct root to be

$\beta=i \sqrt{\left(1-\varepsilon_{0} \mu_{0} v^{2}\right)}$

where the negative root is discarded as physically unrealizable. The propagation velocity of electromagnetic waves in free space is

$v_{0}=\frac{1}{\sqrt{\varepsilon_{0} \mu_{0}}}$

Substituting from Eq. (15) into Eq. (14) yields

$\beta=i \sqrt{\left(1-\left(\frac{v}{v_{0}}\right)^{2}\right.}$

The ratio of acoustic wave velocity to light wave velocity squared is of the order $10^{10}$ and gives $\beta \cong 1 i$.

The amplitude ratio $C_{2} / C_{3}$ is determined from Eq. (13) to be

$$
\frac{C_{2}}{C_{3}}=i \frac{1}{\sqrt{\left(1-\varepsilon_{0} \mu_{0} v^{2}\right)}}=-\frac{1}{\beta} .
$$

Substituting from Eq. (19) into Eqs. (13), the electric fields outside the crystal $\left(x_{2}>0\right)$ become

$$
\left(E_{1}^{+}, E_{2}^{+}, E_{3}^{+}\right)=k\left(C_{1},-\frac{C_{3}}{\beta}, C_{3}\right) \exp \left[i\left\{k\left(\beta x_{2}+x_{3}\right)-\omega t\right\}\right] \text {, }
$$

Accompanying the electric field in this region is a magnetic field found by using Maxwell's curl equation for free space. Therefore, from Eq. (2) 1 and Eqs. (20), the magnetic fields outside $\left(x_{2}>0\right)$ the material become:

$\left(H_{1}^{+}, H_{2}^{+}, H_{3}^{+}\right)=\left(F_{1} C_{3}, F_{2} C_{1},-F_{2} \beta C_{1}\right) \exp \left[i\left\{k\left(\beta x_{2}+x_{3}\right)-\omega t\right\}\right]$,

where

$$
F_{1}=-\frac{i \omega}{\mu_{0} v^{2}} \frac{\varepsilon_{0} \mu_{0} v^{2}}{\sqrt{1-\varepsilon_{0} \mu_{0} v^{2}}}, \quad F_{2}=\frac{i \omega}{\mu_{0} v^{2}} .
$$

It should be remarked that for uncoupled transverse mode $C_{3}=0$. Thus Eqs. (20) yield

$$
E_{1}^{+}=k C_{1} \exp \left[i\left\{k\left(\beta x_{2}+x_{3}\right)-\omega t\right\}\right]
$$

and Eqs. (21) yield

$$
\left(H_{2}^{+}, H_{3}^{+}\right)=\left(F_{2} C_{1},-F_{2} \beta C_{1}\right) \exp \left[i\left\{k\left(\beta x_{2}+x_{3}\right)-\omega t\right\}\right],
$$

Therefore, for the external electromagnetic fields, the transverse mode is represented by Eqs. (22) and (23).

Moreover, for the Rayleigh mode $C_{1}=0$, Eqs. (20) and Eqs. (21) reduce to the following system.

$$
\begin{aligned}
& \left(E_{2}^{+}, E_{3}^{+}\right)=k\left(-\frac{C_{3}}{\beta}, C_{3}\right) \exp \left[i\left\{k\left(\beta x_{2}+x_{3}\right)-\omega t\right\}\right], \\
& \left(H_{1}^{+}\right)=\left(F_{1} C_{3}\right) \exp \left[i\left\{k\left(\beta x_{2}+x_{3}\right)-\omega t\right\}\right] .
\end{aligned}
$$

\section{Rayleigh Mode of piezoelectric materials}

A solution of the Rayleigh mode consists of satisfying the secular equation found from Eqs. (14) and the boundary conditions associated with this mode. The secular equation for the Rayleigh mode with the phase velocity $v$ ( where $v=\omega / k)$ is the expanded coefficient determinant of Eq. (12). Expansion of the coefficient determinant yields an algebraic equation of order six with real coefficients. The six roots of this equation are either real or complex conjugate pairs. Only the complex roots with negative imaginary parts allow Rayleigh surface waves to exist. Therefore, from the total six roots, only three of them are related to Rayleigh surface waves. These roots which concern to Rayleigh surface wave propagation may occur in different forms giving different types of solutions [26].

In view of Rayleigh mode in piezoelectric materials has the coupling of the electromagnetic field and the elastic wave by the piezoelectric constants. Therefore, the secular equation which found by the expanding Eq. (11) becomes an algebraic equation of the sixth order with real coefficients. The three roots are used to obtain the assumed solution as a linear combination

The three acceptable roots are used to obtain the assumed solution as linear combination in the form:

$$
\begin{aligned}
& u_{2}=\sum_{j}\left(A_{2}\right)_{j} \exp \left[i\left\{k\left(\alpha_{j} x_{2}+x_{3}\right)-\omega t\right\}\right], \\
& u_{3}=\sum_{j}\left(A_{3}\right)_{j} \exp \left[i\left\{k\left(\alpha_{j} x_{2}+x_{3}\right)-\omega t\right\}\right], \\
& E_{2}=\sum_{j} k\left(C_{2}\right)_{j} \exp \left[i\left\{k\left(\alpha_{j} x_{2}+x_{3}\right)-\omega t\right\}\right], \\
& E_{3}=\sum_{j} k\left(C_{3}\right)_{j} \exp \left[i\left\{k\left(\alpha_{j} x_{2}+x_{3}\right)-\omega t\right\}\right]
\end{aligned}
$$

where $j=1,2,3 \cdot x_{2} \leq 0$ and the amplitude ratios are defined as:

$N_{j}=\frac{\left(A_{3}\right)_{j}}{\left(A_{2}\right)_{j}}, D_{j}=\frac{\left(C_{2}\right)_{j}}{\left(A_{2}\right)_{j}}, P_{j}=\frac{\left(C_{3}\right)_{j}}{\left(A_{2}\right)_{j}}, j=1,2,3$

which can be found from equation (12) as follows:

$N_{j}=\frac{\left(A_{3}\right)_{j}}{\left(A_{2}\right)_{j}}=-\frac{1}{\Delta_{j}}\left|\begin{array}{lll}a_{11} & a_{12} & a_{13} \\ a_{21} & a_{22} & a_{23} \\ a_{31} & a_{32} & a_{33}\end{array}\right|$, 


$$
\begin{aligned}
D_{j} & =\frac{\left(C_{2}\right)_{j}}{\left(A_{2}\right)_{j}}=\frac{1}{\Delta_{j}}\left|\begin{array}{lll}
L_{11} & L_{12} & L_{13} \\
L_{21} & L_{22} & L_{23} \\
L_{31} & L_{32} & L_{33}
\end{array}\right|, \\
P_{j} & =\frac{\left(C_{3}\right)_{j}}{\left(A_{2}\right)_{j}}=-\frac{1}{\Delta_{j}}\left|\begin{array}{lll}
P_{11} & P_{12} & P_{13} \\
P_{21} & P_{22} & P_{23} \\
P_{31} & P_{32} & P_{33}
\end{array}\right|, \\
\Delta_{j} & =\left|\begin{array}{lll}
G_{11} & G_{12} & G_{13} \\
G_{21} & G_{22} & G_{23} \\
G_{31} & G_{32} & G_{33}
\end{array}\right| .
\end{aligned}
$$

Where the elements of the previous determinants in Eqs. (27) are defined in Appendix A.

The amplitude ratios (29) can be applied to express the solutions in terms of the amplitude constants $\left(A_{2}\right)_{j}$ as:

$$
\begin{aligned}
\left\{u_{2}, u_{3}\right\}= & \sum_{j}\left\{\left(A_{2}\right)_{j}, N_{j}\left(A_{2}\right)_{j}\right\} \times \\
& \exp \left[i\left\{k\left(\alpha_{j} x_{2}+x_{3}\right)-\omega t\right\}\right], \\
\left\{E_{2}, E_{3}\right)= & \sum_{j} k\left\{D_{j}\left(A_{2}\right)_{j}, P_{j}\left(A_{2}\right)_{j}\right\} \times \\
& \exp \left[i\left\{k\left(\alpha_{j} x_{2}+x_{3}\right)-\omega t\right\}\right],
\end{aligned}
$$$$
j=1,2,3, x_{2} \leq 0 \text {. }
$$

\section{The boundary conditions}

Boundary conditions for piezoelectric materials required the following:

(i) A stress free surface, i.e.

$$
T_{2}(0)=T_{4}(0)=0
$$

(ii) Tangent component of the electric field $E_{3}$ must be continuous at $x_{2}=0$, i.e.

$E_{3}^{+}(0)=E_{3}^{-}(0)$.

(iii) Component of the electric displacement must be continuous at $x_{2}=0$, i.e.

$$
D_{2}^{-}(0)=\varepsilon_{0} E_{2}^{+}(0) \text {. }
$$

Applying the boundary conditions given by Eqs. (32)-(34) can be summarized by the following homogeneous system:

$$
\left|\begin{array}{lll}
b_{11} & b_{12} & b_{13} \\
b_{21} & b_{22} & b_{23} \\
b_{31} & b_{32} & b_{33}
\end{array}\right|\left|\begin{array}{l}
\left(A_{2}\right)_{1} \\
\left(A_{2}\right)_{2} \\
\left(A_{2}\right)_{3}
\end{array}\right|=0 \text {. }
$$

This system requires the coefficient determinant to be zero for a nontrivial solution,

$$
\left|\begin{array}{lll}
b_{11} & b_{12} & b_{13} \\
b_{21} & b_{22} & b_{23} \\
b_{31} & b_{32} & b_{33}
\end{array}\right|=0,
$$

Equation (36) is called the secular equation or sometimes called the dispersion equation where

$$
\begin{aligned}
& b_{1 j}=\left(\alpha_{j} C_{11}-C_{14}\right)+\left(C_{13}-\alpha_{j} C_{14}\right) N_{j}-i e_{22} D_{j}-i e_{31} P_{j} \\
& b_{2 j}=\left(C_{44}-\alpha_{j} C_{14}\right)+c_{44} \alpha_{j} N_{j}-i e_{15} D_{j} \\
& b_{3 j}=i\left(e_{22} \alpha_{j}+e_{15}\right)+i e_{15} \alpha_{j} N_{j}+\varepsilon_{22} D_{j}-\left(\frac{i \varepsilon_{0}}{\sqrt{1-\varepsilon_{0} \mu_{0} v^{2}}}\right) P_{j}
\end{aligned}
$$

Numerical procedure must be used to determine the values of $\alpha_{1}, \alpha_{2}, \alpha_{3}$ and $v$ that simultaneously satisfy the coefficient determinants of Eq. (12) and Eq. (36).
Assign values to the speed of the phase $\mathrm{v}$ and the constants $\alpha_{1}, \alpha_{2}, \alpha_{3}$ to investigate transactions determinants in equations (12), (36). The latest format of the solutions may be written as:

$$
\begin{aligned}
& u_{2}=\left(A_{2}\right)_{1}\left(e^{i \alpha_{1} k x_{2}}+M_{21} e^{i \alpha_{2} k x_{2}}+M_{31} e^{i \alpha_{3} k x_{2}}\right) e^{i\left(k x_{3}-\omega t\right)}, \\
& u_{3}=N_{1}\left(A_{2}\right)_{1}\left(e^{i \alpha_{1} k x_{2}}+N_{21} e^{i \alpha_{2} k x_{2}}+N_{31} e^{i \alpha_{3} k x_{2}}\right) e^{i\left(k x_{3}-\omega t\right)}, \\
& E_{2}=k D_{1}\left(A_{2}\right)_{1}\left(e^{i \alpha_{1} k x_{2}}+D_{21} e^{i \alpha_{2} k x_{2}}+D_{31} e^{i \alpha_{3} k x_{2}}\right) e^{i\left(k x_{3}-\omega t\right)}, \\
& E_{3}=k P_{1}\left(A_{2}\right)_{1}\left(e^{i \alpha_{1} k x_{2}}+P_{21} e^{i \alpha_{2} k x_{2}}+P_{31} e^{i \alpha_{3} k x_{2}}\right) e^{i\left(k x_{3}-\omega t\right)}, \\
& E_{1}=u_{1}=0, \quad x_{2} \leq 0 .
\end{aligned}
$$

The amplitude ratios $M_{j 1}, N_{j 1}, D_{j 1}$ and $P_{j 1}$ are determined using the same method used before, and the determinant in Eq. (36) can be written as:

$$
\begin{aligned}
& M_{21}=\frac{\left(A_{2}\right)_{2}}{\left(A_{1}\right)_{1}}=-\frac{1}{\Delta}\left(b_{11} b_{23}-b_{21} b_{13}\right), \\
& M_{31}=\frac{\left(A_{2}\right)_{3}}{\left(A_{2}\right)_{1}}=\frac{1}{\Delta}\left(b_{11} b_{22}-b_{12} b_{21}\right)
\end{aligned}
$$

Where:

$\Delta=\left(b_{12} b_{23}-b_{22} b_{13}\right)$, and the other relations can be found as follows:

$$
\begin{gathered}
N_{j 1}=\left(\frac{N_{j}}{N_{1}}\right) M_{j 1}, D_{j 1}=\left(\frac{D_{j}}{D_{1}}\right) M_{j 1}, P_{j 1}=\left(\frac{P_{j}}{P_{1}}\right) M_{j 1}, \\
\text { 12. Numerical Results and } \\
\text { Discussion }
\end{gathered}
$$

\section{Numerical Results and Discussion}

The numerical solution involves finding the roots of the secular Eq. (11) for various values of propagation velocity. These sets of values and three decay constants are substituted into Eq. (37) to find which set simultaneously satisfied both the secular equation and the boundary conditions. The necessary values of elastic constants, piezoelectric constants and permittivities for Barium Titanate $\left(\mathrm{BaTiO}_{3}\right)$ are given in table 1.

Table 1. Physical properties of $\mathrm{BaTiO}_{3}$ [23]

$$
\begin{array}{ll}
c_{11}=2.73 \times 10^{11} \mathrm{Nm}^{-2} & c_{12}=1.79 \times 10^{11} \mathrm{Nm}^{-2} \\
c_{13}=1.52 \times 10^{11} \mathrm{Nm}^{-2} & c_{33}=1.65 \times 10^{11} \mathrm{Nm}^{-2} \\
c_{44}=5.43 \times 10^{11} \mathrm{Nm}^{-2} & \varepsilon_{\circ}=(1 / 36 \pi) \times 10^{-9} \mathrm{MKS} \\
c_{66}=1.13 \times 10^{11} \mathrm{Nm}^{-2} & e_{15}=21.3 \mathrm{C}^{2} \mathrm{~m}^{2} \\
e_{33}=3.64 \mathrm{C} / \mathrm{m}^{2} & e_{31}=-1.65 \mathrm{C}^{2} \mathrm{~m}^{2} \\
\varepsilon_{11}=1.744 \times 10^{-11} \mathrm{~F} / \mathrm{m} & \rho=6.02 \times 10^{3} \mathrm{Kg} \mathrm{m}^{-3} \\
\varepsilon_{33}=97 \times 10^{-11} \mathrm{~F} / \mathrm{m} &
\end{array}
$$

The frequency equations have been solved to obtain the velocity of the Rayleigh wave propagation and the decay constants which they have been used to calculate the components of the displacement, the electric field.

After obtaining analytical solutions, the numerical computations were represented on the curves in order to facilitate the discussion. The results have been presented graphically to illustrate the nondimensional components of the displacement $u_{2}^{*}=\frac{u_{2}}{u_{2(0)}}$ and $u_{3}^{*}=\frac{u_{3}}{u_{3(0)}}$ and the 
electric field $E_{2}^{*}=\frac{E_{2}}{E_{2(0)}}$ and $E_{3}^{*}=\frac{E_{3}}{E_{3(0)}}$ versus the depth of penetration measured in wavelengths $\mathrm{d}$, where $u_{2(0)}$ and $E_{2(0)}$ are the values of the displacement and the electric field in the $x_{2}$ - direction at the material surface, respectively. The results are complex numbers for the variation of the relative depth $\mathrm{d}$.

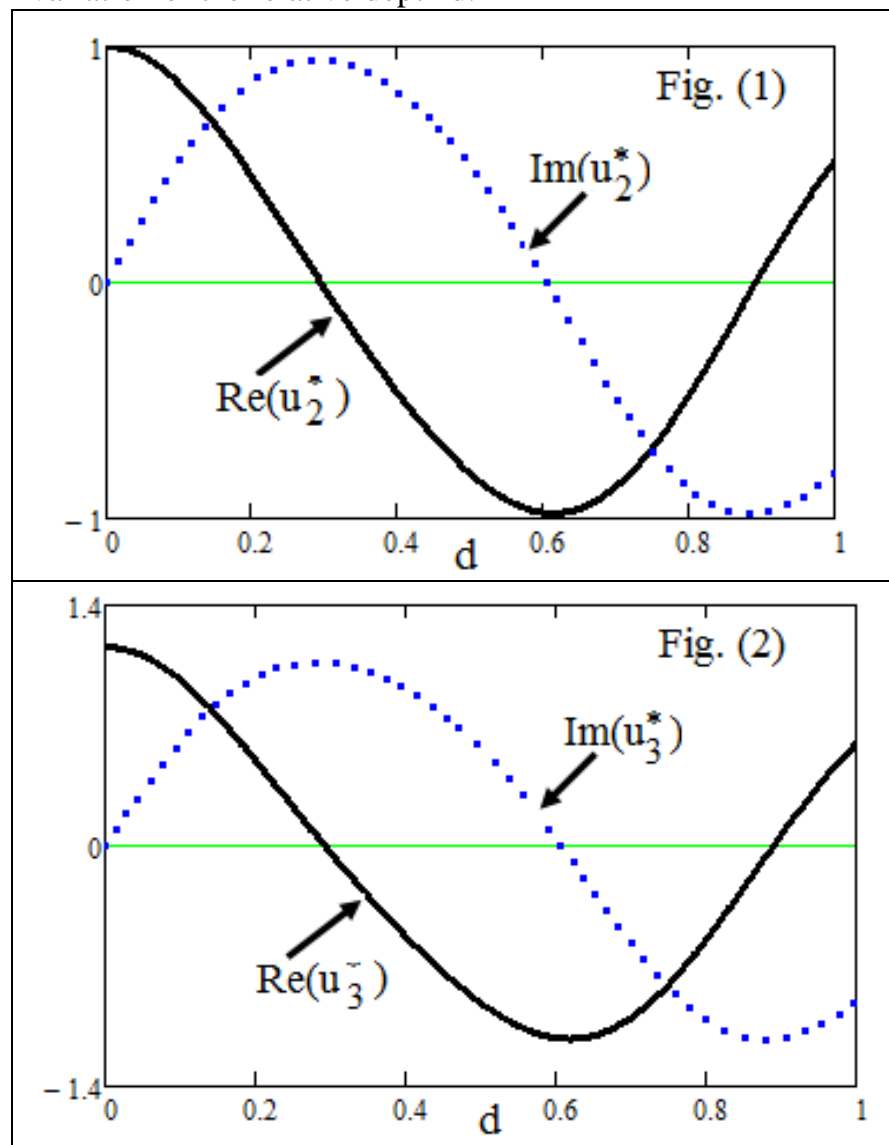

Figures (1) and (2) show the real and imaginary parts of the nondimensional displacement components $u_{2}^{*}$ and $u_{3}^{*}$ versus the relative depth $\mathrm{d}$.

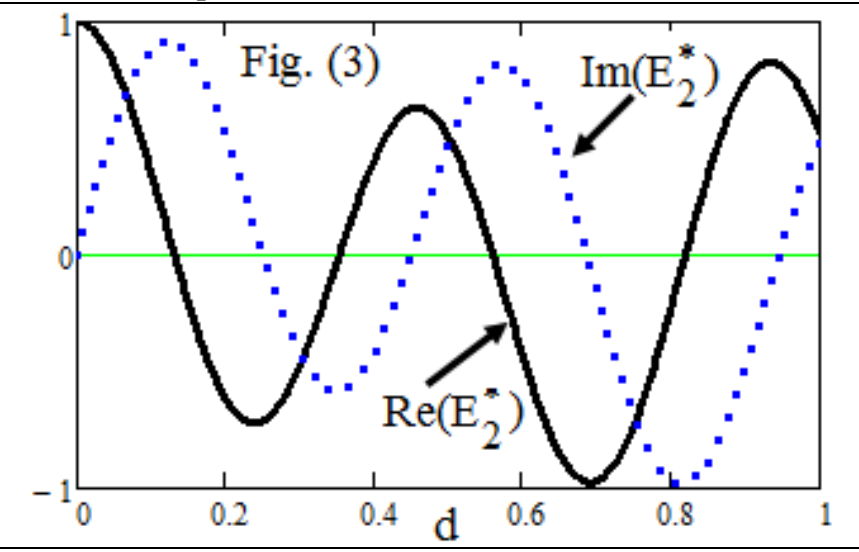

The graphs (1) and (2) give information about the variation of the amplitude ratios of the displacements as a function of relative depth d. While the Figures (3) and (4) present the variation of the amplitude ratios of the electric field versus d. As can be seen, from the Figures there were different trends for the oscillatory behavior of real and imaginary values of these compounds with increasing relative depth.
This fluctuation is a big in the case of the electric field components than in the case of relative displacement components. Furthermore, It is noticed that the electric field of the piezoelectric surface wave exists outside the material as well as inside.

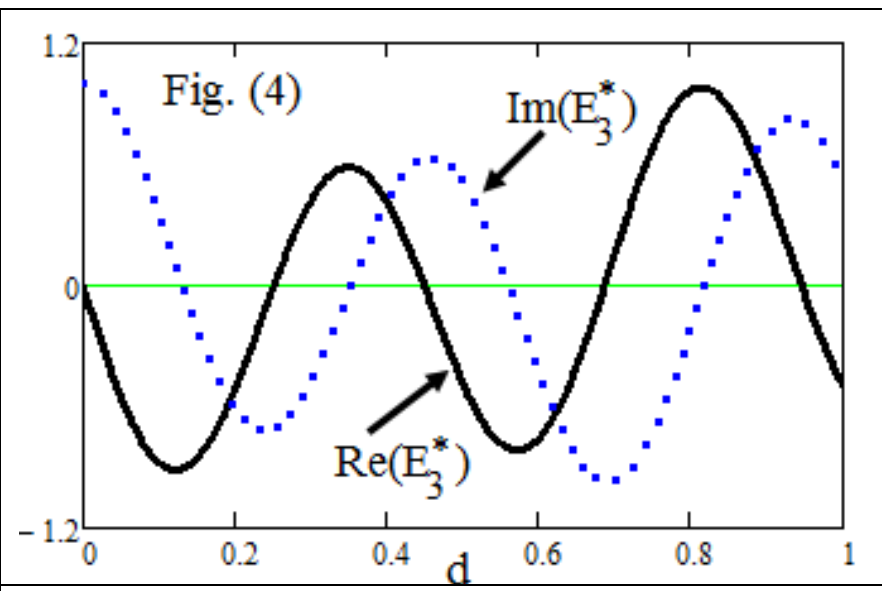

Figures (3) and (4) show the real and imaginary parts of the nondimensional displacement components $E_{2}^{*}$ and $E_{3}^{*}$ versus the relative depth d.

\section{Acknowledgment}

The authors gratefully acknowledge for the deanship of the scientific research of Jazan University with the cooperation of the company of SABIC (Saudi Basic Industries Corporation), Saudi Arabia for supporting this work by (Project No. 23/G5/36).

\section{References}

[1] Rayleigh L., On waves propagating along the plane surface of an elastic solid, Proc. Roy. Soc. Lond. A 17 (1885), pp.4-11.

[2] Jakubik W.P., Surface acoustic wave-based gas sensors, Thin Solid Films, V. 520 (3), (2011), pp.986-993.

[3] Gronewold T.M.A., Surface acoustic wave sensors in the bioanalytical field: Recent trends and challenges, analytica chimica acta 603 (2007) pp.119-128.

[4] Vinh, P.C. and Malischewsky, P.G.; An approach for obtaining approximate formulas for the Rayleigh wave velocity, Wave Motion, 44(7-8), (2007), pp.549-562.

[5] Royer D., A study of the secular equation for Rayleigh waves using the root locus method, Ultrasonics, 39, (2001), pp.223-225.

[6] Abd-alla A.N. and Al-dawy A.A.S., Thermal relaxation times effect on Rayleigh-waves in generalized thermoelastic media, J. of Thermal Stresses, 24, pp. 367-382, (2001)

[7] Abd-alla A.N. and Abo-dahab S.M., Rayleigh waves in magnetothermo-viscoelastic solid with thermal relaxation times, Applied Mathematics and Computations, 149(3), pp.861-877 (2004).

[8] Li, X.F., On approximate analytic expressions for the velocity of Rayleigh waves, Wave Motion, 44(2), (2006), pp.120-127.

[9] Abd-alla A.N. and Asker N., Calculation of bulk acoustic wave propagation velocities in trigonal piezoelectric smart materials, Applied Mathematics and Information Sciences, 8(4), pp.1625-1632 (2014).

[10] Abd-alla A.N. and Asker N., Numerical simulations for the phase velocities and the electromechanical coupling factor of the BleusteinGulyaev waves in some piezoelectric smart materials, Mathematics and Mechanics of Solids, 1081286514527861, first published on April 7, (2014).

[11] Destrade M., Rayleigh waves in symmetry planes of crystals: explicit secular equations and some explicit wave speeds, Mechanics of Materials, 35 (2003), pp.931-939. 
[12] Cristini N.F., Komatitsch D., Carcione J.M., and Cavallini F, Elastic surface waves in crystals. Part 1: Review of the physics, Ultrasonics, 51(6), (2011), pp.653-660.

[13] Vinh P.C. and Linh N.K., An approximate secular equation of generalized Rayleigh waves in pre-stressed compressible elastic solids, International Journal of Non-Linear Mechanics, V.50, (2013), pp.91-96.

[14] Vinh P.C. and Linh N.K., An approximate secular equation of Rayleigh waves propagating in an orthotropic elastic half-space coated by a thin orthotropic elastic layer, Wave Motion, 49 (2012), pp. 681-689.

[15] Murphy J.C. and Destrade M., Surface waves and surface stability for a pre-stressed, unconstrained, non-linearly elastic half-space, International Journal of Non-Linear Mechanics, 44 (2009), pp. 545551.

[16] Abd-alla A.N., Hamdan A.M., Giorgio I. and Del Vescovo D., The mathematical model of reflection and refraction of longitudinal waves in thermo-piezoelectric materials, Archive of Applied Mechanics 84, (2014) 1229-1248.

[17] Abd-alla A.N., Giorgio I., Galantucci L., Hamdan A.M. and del Vescovo D., Wave reflection at a free interface in an anisotropic pyroelectric medium with nonclassical thermoelasticity, Continuum Mechanics and thermodynamics, 28, (2016) 67-84.

[18] Horrillo M.C., Fernandez M.J., Fontecha J.L. et al. ,Optimization of $\mathrm{SAW}$ sensors with a structure $\mathrm{ZnO}-\mathrm{SiO}_{2}-\mathrm{Si}$ to detect volatile organic compounds, Sensors and Actuators B: Chemical, 118 (2006), pp.356361.

[19] Krishnamoorthy S., Iliadis A.A., Beic T. et al., ,An interleukin-6 $\mathrm{ZnO} / \mathrm{SiO}_{2} / \mathrm{Si}$ surface acoustic wave biosensor, Biosensor and Bioelectronics, 24 (2008), pp. 313-318.

[20] Abd-alla A.N., Nonlinear constitutive equations for thermoelectroelastic materials, Mechanics Research Communications, 24 (3), (1999) 335-346.

[21] Abd-alla A.N, and Maugin G.A., Nonlinear magnetoacoustic equations, J. Acoust. Soc. Am. 82, (1978)1746-1752.

[22] Maugin G.A., Continuum Mechanics of Electromagnetic Solid, North-Holland, Amsterdam, (1988).

[23] Yang J.,; The mechanics of piezoelectric structures, World Scientific Publishing Co., Singapore (2008).

[24] Ye Z.G., Handbook of dielectric, piezoelectric and ferroelectric materials Synthesis, properties and applications, Woodhead Publishing Limited, CRC Press, New York (2008).

[25] Topolov V.Y. and Bowen C.R., "Electromechanical Properties in Composites Based on Ferroelectrics", Springer-Verlag London (2009).

[26] Chirita S., Ciarletta M. and Tibullo V., Rayleigh surface waves on a Kelvin-Voigt viscoelastic half-space. J. Elast. 115, 61-76 (2014).

[27] Sharma M.D., Propagation and attenuation of Rayleigh waves in generalized thermoelastic media, Journal of Seismology, 18 (1), (2014) 61-79.

[28] Bucur,A.V., Passarella F. and Tibullo V., Rayleigh surface waves in the theory of thermoelastic materials with voids. Meccanica 49, (2014) 2069-2078.

[29] Torello D., Thiele S., Matlack K.H., Kim J.Y., Qu J. and Jacobs, L.J., Diffraction, attenuation, and source corrections for nonlinear Rayleigh wave ultrasonic measurements, Ultrasonics, 56, (2015) 417-426 .

[30] Lin C.I., Lu Y., He C., Song G.C.H. and Lee Y.C., Cylindrical Rayleigh surface waves on a layer-coated cylinder measured by PVDF transducer and defocusing measurement method, NDT \& E International, 74, (2015), 43-49.

[31] Singh, B., Sindhu, R. and Singh, J., Rayleigh wave in a micropolar thermoelastic medium without energy dissipation, Engineering Solid Mechanics, 4 (1), (2016) 11-16.

\section{Appendix A.}

$$
\begin{aligned}
& a_{11}=-v^{2} \rho+C_{11} \alpha_{j}^{2}-2 C_{14} \alpha_{j}+C_{44}, \quad a_{13}=i e_{31} \alpha_{j}, \\
& a_{12}=i\left(e_{22} \alpha_{j}+e_{15}\right), \quad a_{21}=-C_{14} \alpha_{j}^{2}+C_{44} \alpha_{j}+C_{13} \alpha_{j}, \\
& a_{22}=i e_{15} \alpha_{j}, \quad a_{23}=i e_{33}, \quad a_{31}=i \mu_{0} v^{2}\left(e_{22} \alpha_{j}+e_{15}\right), \\
& a_{32}=\mu_{\circ} \varepsilon_{11} v^{2}-1, \quad a_{33}=\alpha_{j}
\end{aligned}
$$

$$
\begin{aligned}
& L_{11}=-v^{2} \rho+C_{11} \alpha_{j}^{2}-2 C_{14} \alpha_{j}+C_{44}, \quad L_{23}=i e_{33}, \\
& L_{12}=C_{13} \alpha_{j}-C_{14} \alpha_{j}^{2}+C_{44} \alpha_{j}, \quad L_{13}=i e_{31} \alpha_{j}, \quad L_{33}=\alpha_{j} \\
& L_{22}=-v^{2} \rho+C_{44} \alpha_{j}^{2}+C_{33}, \quad L_{31}=i \mu_{0} v^{2}\left(e_{22} \alpha_{j}+e_{15}\right), \\
& L_{21}=-C_{14} \alpha_{j}^{2}+C_{44} \alpha_{j}+C_{13} \alpha_{j}, \quad L_{32}=i e_{15} v^{2} \mu_{0} \alpha_{j},
\end{aligned}
$$

and also:

$$
\begin{aligned}
& P_{11}=-v^{2} \rho+C_{11} \alpha_{j}^{2}-2 C_{14} \alpha_{j}+C_{44}, \quad P_{23}=i e_{15} \alpha_{j}, \\
& P_{12}=C_{13} \alpha_{j}-C_{14} \alpha_{j}^{2}+C_{44} \alpha_{j}, \quad P_{13}=i\left(e_{22} \alpha_{j}+e_{15}\right), \\
& P_{21}=-C_{14} \alpha_{j}^{2}+C_{44} \alpha_{j}+C_{13} \alpha_{j}, \quad P_{32}=\mu_{0} \varepsilon_{11} v^{2}-1, \\
& P_{22}=-v^{2} \rho+C_{44} \alpha_{j}^{2}+C_{33}, \quad P_{33}=\alpha_{j}, \\
& P_{31}=i \mu_{0} v^{2}\left(e_{22} \alpha_{j}+e_{15}\right), \\
& G_{11}=C_{13} \alpha_{j}-C_{14} \alpha_{j}^{2}+C_{44} \alpha_{j}, \quad G_{12}=i\left(e_{22} \alpha_{j}+e_{15}\right), \\
& G_{13}=i e_{31} \alpha_{j}, \quad G_{21}=-v^{2} \rho+C_{44} \alpha_{j}^{2}+C_{33}, \\
& G_{22}=i e_{15} \alpha_{j}, \quad G_{23}=i e_{33}, \quad G_{31}=i e_{15} v^{2} \mu_{o} \alpha_{j}, \\
& G_{32}=\mu \varepsilon_{11} v^{2}-1, \quad G_{33}=\alpha_{j} .
\end{aligned}
$$

\begin{tabular}{|c|l|}
\hline \multicolumn{2}{|c|}{ Appendix B } \\
\hline$T_{i j}$ & is the stress tensor \\
\hline$S_{k \ell}$ & is the strain tensor \\
\hline$e_{i k \ell}$ & is the piezoelectric tensor \\
\hline$c_{i j k \ell}$ & is the stiffness tensor \\
\hline$\varepsilon_{i k}$ & is the symmetric permittivity tensor \\
\hline$\varepsilon_{i j k}$ & is the rotation tensor \\
\hline$u_{i}$ & is the displacement tensor \\
\hline$D_{i}$ & is the component of electric displacement, \\
\hline$E_{i}$ & is the electric field \\
\hline$H_{j}$ & is the magnetic field intensity, \\
\hline$\rho$ & is the mass density of the elastic medium \\
\hline$\mu_{\circ}$ & is the permeability of field space \\
\hline$\varepsilon_{\circ}$ & is the permittivity of free space \\
\hline
\end{tabular}

